\section{P63 Evaluation of a Family-Focused, Garden- Based, Nutrition Education Program: The Growing Gardens, Growing Health Program}

ChengLi, MS, cl852@scarletmail.rutgers.edu, Rutgers University, Department of Nutritional Sciences, 11 Suydam Street, 2nd Floor, New Brunswick, NJ 08901; Heather Peracchio, MS, RDN, CDN, University of Connecticut, Department of Extension; Kaitlin Latham, BA, Norwalk Health Department; Theresa Argondezzi, MPH, CHES, CPH; Michael Puglisi, PhD, RD, University of Connecticut, Department of Nutritional Sciences;

Debra Palmer-Keenan, PhD, Rutgers University, Department of Nutritional Sciences

Background (Background, Rationale, Prior Research, and/or Theory): Garden-based nutrition education programs are viewed as a promising strategy to promote nutrition and physical activity for families.

Objective: To evaluate the impact of a 10-week, familyfocused, garden-based, nutrition education program.

Study Design, Setting, Participants, Intervention: The Growing Gardens, Growing Health program was a 10week program that promoted healthier lifestyles to families through gardening. It was implemented by University of Connecticut's Expanded Food and Nutrition Education Program (EFNEP), the Norwalk Health Department, two Certified Master Gardener volunteers, and a Rutgers PhD student. Each 90-minute session included a Master Gardener designed gardening component and a Registered Dietitian designed nutrition education/cooking component. During 2017, 29 families were recruited through flyers and emails.

Outcome Measures and Analysis: Parent pre/postintervention surveys assessed their: fruit and vegetable (FV) consumption; physical activity (PA) behaviors, selfefficacy and readiness to change FV consumption, PA and gardening behaviors; and their children's FV consumption. Post-program satisfaction was also assessed. Descriptive statistics and Wilcoxon signed-rank test were performed using SAS 9.4.

Results: Of the parents $(n=17)$ who completed the program, $82 \%$ indicated they were extremely satisfied with the program and 94\% would strongly recommend the program. Significant increases in parents' median confidence levels regarding FV gardening were noted $(Z=18$, $P=$.0078). Although parents' reported FV consumption didn't increase significantly, their confidence in consuming at least five servings of FV daily did $(Z=14, P=.0156)$. For those who cook with fresh FV $<4$ times/week preintervention, 50\% cooked more at post-intervention. Among those whose children who ate FV $<2$ times/day, $62.5 \%$ reported that their children were eating more fruits, and $45 \%$ reported their children were eating more vegetables, post-intervention. Parents' readiness to exercise for at least 30 minutes/day on most days also increased $(Z=14$, $P=.0156)$.

Conclusions and Implications: This family-focused, garden-based nutrition education program may have the potential to increase participants FV intake and PA behaviors. Further research is needed to identify if a longer intervention would result in additional improvements. Funding: Campbell's Healthy Communities Program.

\section{P64 Evaluation of Worksite Wellness Nutrition and Physical Activity Interventions and Their Subsequent Impact on Participants' Body Compositions: A Systematic Review}

Victoria Sandercock, BS, Eastern Illinois University; Jeanette Andrade, PhD, LDN, RDN, jmandrade@eiu.edu, Eastern Illinois University, 600 Lincoln Avenue, Charleston, IL 61920

Background (Background, Rationale, Prior Research, and/or Theory): Worksite wellness programs have shifted from preventing injuries to reducing or preventing employee obesity. These programs include both nutrition education and physical activity, yet results are inconsistent in reducing weight.

Objective: To evaluate worksite wellness nutrition and physical activity interventions and their subsequent impact on participants' body composition.

Study Design, Setting, Participants, Intervention: Articles, between 01-1980 and 11-2017, were extracted from Pub- Med, CINAHL, SCOPUS, and PsycINFO databases using the key words, "worksite wellness nutrition and physical activity interventions/programs" and "weight". A 9-point inclusion and an 8-point exclusion criteria were established. Two researchers independently extracted articles, applied inclusion/exclusion criteria to titles and abstracts and assessed the quality of articles using the Academy of Nutrition and Dietetics Evidence Analysis Manual.

Outcome Measures and Analysis: Participants' body compositions included weight, BMI, fat free mass, and waist circumference.

Results: A total of 962 articles were identified, of which 51 articles were fully examined after the inclusion/ exclusion criteria and 23 articles were included in the study. Eleven studies resulted in significant decreases in body composition $(P<.05), 6$ studies resulted in body composition decreases without statistical significance $(P>.05)$ and 6 studies did not show any reductions in body composition. Interventions ranged from daily, weekly, or monthly educational sessions via traditional (e.g. face-to-face) or online settings and were led by registered dietitians or nutritionists and exercise physiologists. Interventions that resulted in larger reductions of body composition, regardless of time, held educational sessions in a traditional setting and incorporated a self-driving theory such as motivational interviewing, self-determination, or health belief. Conclusions and Implications: The evidence supports that worksite wellness nutrition and physical activity interventions that include traditional education settings with expert educators and a self-driving theory will aid in reducing participants' body compositions. Effectively this may aid corporate dietitians in establishing interventions that improve employees' overall health.

Funding: None. 


\section{P65 Factors Influencing Childcare Providers' Engagement of Parents Regarding Child Nutrition \\ Aileen Garcia, MA, aileen.garcia@huskers.unl.edu, University of Nebraska-Lincoln, 135 Mabel Lee Hall, Lincoln, NE 68588; Dipti Dev, PhD; Virginia Stage, PhD, $L D N, R D N$, East Carolina University}

Background (Background, Rationale, Prior Research, and/or Theory): The Academy of Nutrition and Dietetics, parents, childcare directors, and health consultants agree that nutrition education is instrumental to improving children's and families' nutrition knowledge and eating behavior (Benjamin Neelon \& Briley, 2011; Gupta et al., 2005). More recent research, however, has demonstrated that childcare providers often report challenges to engaging parents in topics about their children's nutrition (Lyn et al., 2014; Stage et al., 2017).

Objective: To determine the factors influencing childcare providers' engagement with parents regarding child nutrition in childcare centers (CCCs) and family childcare homes (FCCHs).

Study Design, Setting, Participants, Intervention: This was a cross-sectional, quantitative study on CCCs and FCCHs in Nebraska. CCC directors $(\mathrm{n}=337)$ and FCCH providers $(n=1,153)$ completed self-administered surveys.

Outcome Measures and Analysis: The dependent variable was parent engagement. Fifteen variables were examined as predictors for parent engagement-barriers to communication, participation in Go Nutrition and Physical Self- Assessment in Child Care (Go NAP SACC), National Association for the Education of Young Children (NAEYC) accreditation, participation in Quality Ratings and Improvement Systems (QRIS), feeding practices, professional development regarding child nutrition, and child-provider ratio. Structural equation modeling was conducted to examine the relation between variables for CCCs and for FCCHs.

Results: For CCC's, NAEYC accreditation, providers' barriers regarding parents' cultural beliefs about food that are not always consistent with healthy eating, parents not liking the taste of healthy foods, and parents prioritizing other food related topics (allergies or children's food intake) significantly predicted parent engagement. For FCCHs, participation in Go NAP SACC, perceiving parents as busy, not wanting to offend parents, and practicing family style dining were significantly related to parent engagement. For both CCCs and FCCHs, professional development regarding child nutrition was related to parent engagement.

Conclusions and Implications: Focusing professional development on childcare contexts and addressing providers' barriers may improve parent engagement.

Funding: USDA.

\section{P66 Family Nutrition Education Improves Healthy Eating and Preferences, but Children and Adults Differ in Behavioral Changes}

Elizabeth Miller, $\mathrm{MPH}, \mathrm{CPH}$, Common Threads; Natalie Goldsworthy, MS,

ngoldsworthy@commonthreads.org, Common Threads,
3811 Bee Caves Road, Suite 108, Austin, TX 78746; Allison Wojtowicz, MA; Neilé Edens, PhD

Objective: Nutrition education and cooking interventions are used to promote healthy habits among children and their families. The purpose of this research is to evaluate the effect of a Family Cooking Class (FCC) on participant vegetable consumption, attitudes, and behaviors. Target Audience: Families with children, grades 3-8, in schools where at least $80 \%$ of students were eligible for free or reduced priced lunch totaling 1,375 children and 757 adults over two years.

Theory, Prior Research, Rationale: FCC is grounded in the socio-ecological model and designed to increase knowledge and skills about healthy cooking and eating in the whole family unit.

Description: FCC is composed of six 2-hour lessons incorporating nutrition education and hands-on cooking and is facilitated by a trained instructor. Nutrition education is provided on healthy ingredient substitutions and balanced meal planning while the cooking component focuses on cost saving methods and recipes. Families work together each lesson to cook 2-4 healthy dishes which are eaten together at the end of the class.

Evaluation: Evaluation of child and adult outcomes were conducted using a quasi-experimental pre-post survey design between 2015 and 2017. A convenience sample of 108 students and 90 adults were included in analysis, representing 24 FCCs from 5 markets. FCC was found to significantly improve child vegetable liking $(P=.001)$, exposure $(P<.001)$, and consumption $(P<.001)$ as well as adult vegetable consumption $(P=.001)$. Children also reported increased cooking self-efficacy $(P<.001)$, frequency cooking at home $(P=.005)$, and telling family about healthy eating $(P=.002)$. Adults agreed that their child's cooking skills were improved after programming $(P<.001)$.

Conclusions and Implications: FCC shows promise in improving family vegetable consumption which can lead to long term positive health outcomes. However, adults' data did not support all child-reported behavioral outcomes, suggesting that adults may have different perception than children of program efficacy.

Funding: None.

\section{P67 Farm-to-Clinician: A Culinary Medicine Approach to Healthcare Through a Food Systems Lens}

Anna Diffenderfer, MS, RDN, LN, anna.diffenderfer@montana.edu, Montana State University, 349 Reid Hall, Bozeman, MT 59717; D. Coleen R. Kaiser, MS, RDN, LN; Colleen McMilin, PhD, $\mathrm{MPH}$; Marcy Gaston, MS, RDN, LN

Objective: To educate future healthcare professionals about the importance of nutrition in the prevention and treatment of disease, utilizing an experiential approach emphasizing local food systems.

Continued on page S39 